\title{
In situ deposited multilayer integrated hydrogels for deformable and stretchable supercapacitors
}

\author{
Yanfang Ren ${ }^{1}$, Chencheng Sun ${ }^{3}$, Yunlong Liu ${ }^{1}$, Ying Hong ${ }^{2}$, Qian Wang ${ }^{2 *}$, Wenli Zhao ${ }^{2}$, Shuhong Li $^{1^{*}}$, \\ Wenjun Wang ${ }^{1}$ and Xiaochen Dong ${ }^{2 *}$
}

\begin{abstract}
Hydrogel systems promote the development of flexible energy storage devices because of their inherent mechanical elasticity and ionic conductivity. However, achieving stable energy storage capacity under violent mechanical deformation is still a challenge for hydrogel devices. In this work, an all-in-one integrated supercapacitor (AISC) was assembled using in situ deposited polyaniline/graphene oxide nanocomposites for both sides of the incorporated ionic hydrogel electrolyte. The assembly process of the AISC was greatly simplified, and the displacement and separation of the multilayer structured hydrogel complex were avoided during mechanical deformation. The hydrogel electrolyte with ionic additives exhibited strong adhesion and flexibility, and high ionic conductivity, thereby ensuring the excellent specific capacitance and rate performance of the AISC. The specific capacitances of the AISC were $222.8 \mathrm{mF} \mathrm{cm}^{-2}$ at the current density of $0.2 \mathrm{~mA} \mathrm{~cm}^{-2}$ and $151.7 \mathrm{mF} \mathrm{cm}^{-2}$ at $3.2 \mathrm{~mA} \mathrm{~cm}^{-2}$. The capacitance retention rate was $68.1 \%$. The energy density of a piece of the device reached $44.6 \mu \mathrm{Wh} \mathrm{cm}$ at a power density of $120.0 \mu \mathrm{W} \mathrm{cm} \mathrm{cm}^{-2}$. Moreover, reliable and reproducible energy storage was acquired under bending, compression, and stretching deformations. The AISC was also easily assembled in series to power a light-emitting diode (LED) light. This work provides a facile approach to the construction of flexible supercapacitors for the development of energy storage devices in flexible electronics.
\end{abstract}

Keywords: hydrogel electrolyte, flexibility, polyaniline/graphene oxide, supercapacitor

\section{INTRODUCTION}

Flexible electronic devices with prominent scalability and portability have been proposed for use in a wide array of wearable devices, electronic skins, smart textiles, and others $[1,2]$. The development of flexible electronic devices strictly relies on stretchable functional components and energy storage modules. In particular, the design of ductile deformation-tolerant energy storage devices has become a bottleneck in integrated flexible electronics. Flexible supercapacitors (SCs), which feature high power density, fast charge-discharge capability, and long-term cycling stability, show particularly wide application prospects in flexible electronics [3].

Mechanically deformable electrode and electrolyte materials, and structures are the most critical factors in the assembly of flexible devices [4]. The most widely used electrode material in the field of flexible SCs is conductive polymer, which shows high electrical conductivity by the $\pi-\pi$ stacking between polymer chains [5]. During the electrochemical reaction process, conductive polymers can store charges through reversible n-type or p-type ion doping and purification [6]. For example, Oh et al. [7] used an electrode fabricated with porous polyaniline (PANI) composites to assemble flexible SCs. However, the pseudocapacitance characteristics of conductive polymers are observed in limited ion exchange reactions during the charge transfer process, thereby resulting in poor rate performance. Moreover, the serious electrode cracking that occurs during ion doping and purification significantly reduces the stability and reliability of flexible SCs. With the critical requirements of flexible SCs in practical applications, recent studies mainly focused on enhancing the stretchability and rate performance of conductive polymers in flexible SCs [8].

One of the most feasible approaches is to incorporate conductive polymers into appropriate stretchable substrates [9]. Hydrogels, which are porous, stretchable, and hydrophilic polymer networks with high water content, can be utilized in electrode-electrolyte-electrode layered structures and show great promise in stretchable SCs [10]. Hydrogels are commonly applied as integrated separators and electrolytes because of their inherent electronic insulation and ion conductance features, and their enhanced ion conductivity relative to industrialized solid electrolytes [11]. With the appropriate incorporation of active materials, conductive hydrogels can also be utilized as stretchable electrodes in flexible SCs. For example, Yang et al. [12] proposed all-solid SCs with poly(3,4-ethylene dioxythiophene)/ PANI hydrogel electrodes and polyvinyl alcohol/sulfuric acid (PVA/ $\mathrm{H}_{2} \mathrm{SO}_{4}$ ) hydrogel electrolytes. The all-solid SCs showed excellent energy storage performance and exhibited an energy density of $0.25 \mathrm{~mW} \mathrm{~h} \mathrm{~cm}^{-3}$ at a power density of $107.14 \mathrm{~mW} \mathrm{~cm}^{-3}$. However, the sandwich-structured device was

\footnotetext{
${ }^{1}$ Shandong Provincial Key Laboratory of Optical Communication Science and Technology, School of Physical Science and Information Technology, Liaocheng University, Liaocheng 252059, China

${ }^{2}$ Key Laboratory of Flexible Electronics (KLOFE) \& Institute of Advanced Materials (IAM), School of Physical and Mathematical Sciences, Nanjing Tech University (NanjingTech), Nanjing 211800, China

${ }^{3}$ School of Electronic and Information Engineering, Changshu Institute of Technology, Changshu 215500, China

* Corresponding authors (emails: lishuhong@lcu.edu.cn (Li S); chelseawq@njtech.edu.cn (Wang Q); iamxcdong@njtech.edu.cn (Dong X))
} 
found to remain problematic in long-term applications. First, a certain pressure on the device was required along with the charge-discharge process to achieve a close contact between the electrodes and the electrolytes [13]. Second, the delamination and displacement of the laminated structure took place under large deformations, resulting in an obvious decrease in energy storage performance [14]. Therefore, exploiting novel configurations is a top priority for achieving seamless connection and conformal deformation between the electrodes and electrolytes in stretchable SCs. Research has shown that the all-in-one assembly in flexible SCs is an effective way to reduce performance dissipation under mechanical deformation. Hu et al. [15] prepared all-in-one configured hydrogel SCs through a one-step deposition of nanocomposites on both sides of a polyacrylic acid (PAA) hydrogel. The device could work at scanning rates of up to $10 \mathrm{~V} \mathrm{~s}^{-1}$ with reserved characteristics of tension and compression. Guo et al. [16] prepared integrated SCs by depositing PANI and single-walled carbon nanotubes on two layers of $\mathrm{PVA} / \mathrm{H}_{2} \mathrm{SO}_{4}$ hydrogel film; the specific capacitance of the fully configured SCs reached $15.8 \mathrm{mF} \mathrm{cm}^{-2}$ at a current density of $0.044 \mathrm{~mA} \mathrm{~cm}^{-2}$. In addition, a large number of hydrogen bonds provided by the physically crosslinked hydrogel promoted an enviable self-healing property in the device. To further optimize the electrochemical performance of the integrated devices, the authors introduced redox additives into the hydrogel electrolyte. Han et al. [17] designed a redox bromide ion-compensated hydrogel electrolyte (SA-Zn-Br) for flexible $\mathrm{Zn}$ ion hybrid SCs; $\mathrm{SA}-\mathrm{Zn}-\mathrm{Br}$ displayed a maximum energy density of $605 \mathrm{~W} \mathrm{~h} \mathrm{~kg}^{-1}$ at a power density of $1848 \mathrm{~W} \mathrm{~kg}^{-1}$ and superior voltage of $2.6 \mathrm{~V}$. The additional redox reactions at the electrode-electrolyte interface from the incorporated redox additives significantly increased the specific capacitance and energy density of the flexible SCs [18].

Herein, we assembled an all-in-one integrated SC (AISC) via a laminated hydrogel system, in which a PANI/graphene oxide (GO) hydrogel electrode was in situ deposited on both sides of a piece of hydrogel electrolyte (GELE). Ionic additives were incorporated into the hydrogel electrolyte to form multiple crosslinking networks, which greatly improved the mechanical and electrochemical properties of the AISC. The specific capacitances of the fully configured SC were 222.8 and $151.7 \mathrm{mF} \mathrm{cm}^{-2}$ at current densities of 0.2 and $3.2 \mathrm{~mA} \mathrm{~cm}^{-2}$, respectively. The area energy density reached $44.6 \mu \mathrm{W} \mathrm{h} \mathrm{cm}{ }^{-2}$ at the power density of $120.0 \mu \mathrm{W} \mathrm{cm}{ }^{-2}$. In practice, the PANI/GO-GELE device displayed excellent mechanical resistance to bending, compression, and tension with optimized electrochemical performance. With the excellent adhesive properties of the hydrogel, the device can be easily assembled in a series connection to power light-emitting diode (LED) lights.

\section{EXPERIMENTAL SECTION}

\section{Materials}

Acroleic acid (AA, 99\%), acrylamide (AM, 99\%), and $N, N$ methylene bisacrylamide (MBA) were purchased from SigmaAldrich. Sodium alginate (SA), $\mathrm{CuSO}_{4}$, and $\mathrm{CuBr}_{2}$ were obtained from Aladdin Chemical Co., Ltd. Aniline, sulfuric acid $\left(\mathrm{H}_{2} \mathrm{SO}_{4}\right)$, and ammonium persulfate (APS) were purchased from Yonghua Fine Chemical Co., Ltd. The GO suspension was obtained from Zhongke Yueda (Shanghai) Material Technology Co., Ltd. All chemical reagents were of analytical grade and used without further purification.

\section{Preparation of GELE film}

First, $1 \mathrm{~g}$ of $\mathrm{AM}$ was dissolved in $4 \mathrm{~mL}$ of deionized (DI) water, and the resulting mixture was then added with $4.5 \mathrm{~g}$ of SA aqueous solution (5\%) under vigorous stirring. Thereafter, $2 \mathrm{~mL}$ of AA was added to the aforementioned solution. The mixed solution was sonicated for $30 \mathrm{~min}$ to ensure a uniform dispersion. Then, $0.2 \mathrm{~g}$ of APS and $5 \mathrm{mg}$ of MBA were dispersed in a mixture solution via ultrasonic treatment. The mixed solution was injected into a mold and polymerized at $55^{\circ} \mathrm{C}$ for $2 \mathrm{~h}$. The obtained hydrogel precursor (GELEP) was removed from the mold and washed with DI water to remove the unreacted monomer. Finally, $0.48 \mathrm{~g}$ of $\mathrm{CuSO}_{4}$ and $0.08 \mathrm{~g}$ of $\mathrm{CuBr}_{2}$ were dispersed in $20 \mathrm{~mL}$ of DI water. The GELEP film was soaked in the solution for $24 \mathrm{~h}$ to fully achieve material balance. The product was then named GELE and used as the hydrogel electrolyte. The introduction of $\mathrm{Cu}^{2+}$ changed the color of the hydrogel from white to light blue. The size of the GELEP and the immersion time were strictly controlled to ensure the introduction of the same amount of ions.

\section{Preparation and electrochemical characterization of PANI/GO- GELE AISC}

In brief, the PANI/GO-GELE hydrogel complex was obtained by in situ depositing PANI/GO nanocomposites onto the two-sided faces of the GELE film. The electrode materials of aniline and GO were firmly in situ polymerized and deposited onto the two sides of the GELE separator. Solution A was prepared by mixing $1 \mathrm{~mL}$ of aniline and $2 \mathrm{~mL}$ of GO solution $\left(3 \mathrm{mg} \mathrm{mL}^{-1}\right)$ into $2 \mathrm{~mL}$ of $1 \mathrm{~mol} \mathrm{~L}^{-1} \mathrm{H}_{2} \mathrm{SO}_{4}$ solution with constant stirring in an ice water bath for $10 \mathrm{~min}$. Solution B was prepared by dissolving $0.56 \mathrm{~g}$ of APS in $2 \mathrm{~mL}$ of $1 \mathrm{~mol} \mathrm{~L}^{-1} \mathrm{H}_{2} \mathrm{SO}_{4}$ solution cooled at $0^{\circ} \mathrm{C}$. Solutions $\mathrm{A}$ and $\mathrm{B}$ were merged at $0^{\circ} \mathrm{C}$, and the GELE was immersed into the mixture of solutions $\mathrm{A}$ and $\mathrm{B}$ at $-7^{\circ} \mathrm{C}$ for $30 \mathrm{~min}$. After the polymerization, the PANI-decorated PANI/GO-GELE with bilateral PANI/GO electrode materials was obtained. Finally, the all-in-one flexible SC was assembled by connecting both ends of the PANI/GO electrode with carbon cloth as the current collector.

\section{Material characterization}

The crystalline structures of the nanocomposite were profiled by X-ray diffraction (XRD) (SmartLab $3 \mathrm{KW}$ ). The microstructure and elemental analysis of the hydrogel were characterized by scanning electron microscopy (SEM) and energy-dispersive Xray spectroscopy (EDS) (Hitachi, S-4800, Japan). The mechanical properties of the hydrogel were measured using a test platform with a stepping motor (ESM302, Mark-10) and a force gage (M4-2).

\section{Electrochemical characterization}

The electrochemical performance of the AISC was measured by cyclic voltammetry $(\mathrm{CV})$ and galvanostatic charge/discharge (GCD) in a two-electrode configuration by using an electrochemical workstation (Chenhua CHI660E, Shanghai) and a NEWARE battery testing system. The GCD curve measured on the specific capacitance was calculated as follows [19]:

$C=\frac{I \times \Delta t}{S \times \Delta V}$ 
where $I$ is the applied current, $\Delta t$ is the discharge time, $\Delta V$ is the charge-discharge voltage range, and $S$ is the area involved in the chemical reaction.

The energy density $(E)$ and power density $(P)$ of the device were further calculated through the following [20]:

$E=\frac{1}{2} C \Delta V^{2}$,

$P=\frac{E}{\Delta t}$.

\section{RESULTS AND DISCUSSION}

Preparation and characterization of PANI/GO-GELE AISC

The fabrication process of the PANI/GO-GELE hydrogel complex and AISC is presented in Fig. 1. First, the monomers of AA, AM, APS, and MBA were dissolved in the SA solution and copolymerized at $55^{\circ} \mathrm{C}$ for $2 \mathrm{~h}$ to form the hydrogel electrolyte precursor GELEP. Then, the GELEP film was immersed in the $\mathrm{CuSO}_{4} / \mathrm{CuBr}_{2}$ solution for $24 \mathrm{~h}$ to form GELE [21]. In this step, the oxygen-containing polar groups on the molecular chain of $\mathrm{SA}$, including hydroxyl $(-\mathrm{OH})$ and carboxyl $\left(-\mathrm{COO}^{-}\right)$, were easily coupled with the $\mathrm{Cu}^{2+}$ ions under mild conditions. The redundant $\mathrm{Cu}^{2+}$ further coordinated with the carboxyl groups from the PAA chain through ionic interactions [22]. Third, the GELE film was immersed in an acidic solution of aniline and GO nanosheets for in situ deposition. In the acidic environment, PANI was quickly polymerized, thereby forming sufficient hydrogen bonds with oxygen-containing functional groups on the surface of GO, and was then deposited onto the two sides of the GELE film, as reflected by the color changes and crosssectional structure evolution in the images in Fig. S1 [23]. The PANI/GO network established an extensive interaction with the GELE film to reduce the interfacial impedance and ensure rapid charge transfer at the electrode/electrode interface. In particular, the quick deposition of PANI created a strong adhesion to the GELE film through ionic interaction and formed a distinct sandwich structure (Fig. 1b), which also prevented the infiltration of hydrophilic active materials into the electrolyte film and avoided short circuits in the device [24].

The PANI/GO-GELE hydrogel complex incorporated by multiple physical and chemical interactions showed excellent mechanical and electrical properties because of the GELE substrate that interacted with ions. As shown in Fig. S2, the hydrogel substrate, GELEP, and GELE showed remarkable mechanical deformation, which could sustain sufficient flexibility for stretchable SCs.

Fig. 2a shows that the integrated hydrogel device could withstand a weight of $500 \mathrm{~g}$ with negligible damage and revert to its original state after the removal of the external force. More importantly, the PANI/GO-GELE hydrogel complex (Fig. 2b) is easy to stretch in the original, twisted, knotted, and crossover states, thereby exhibiting excellent compression and tension properties. Meanwhile, the hydrogel complex presented good mechanical resistance to compression and quickly returned to its original states after bearing weights of 100,200 , and $500 \mathrm{~g}$ (Fig. 2c). Fig. 2d shows that the hydrogel adhered tightly to the carbon cloth, thereby enabling close contact with the current collector to ensure reliable electrochemical performance in the PANI/GO-GELE AISC.

Tensile tests were conducted to quantitatively assess the stretchability of the hydrogel complex. As shown in Fig. 2e, the stretchability of the hydrogel complex was greatly improved by the hydrogel electrolyte substrate because of the rapid chelating performance of $\mathrm{Cu}^{2+}$ with carboxylates and amino/hydroxyl groups and the synergistic effects of the multiple interacted PANI/GO networks [25]. Fig. 2f shows the XRD spectrum of

a
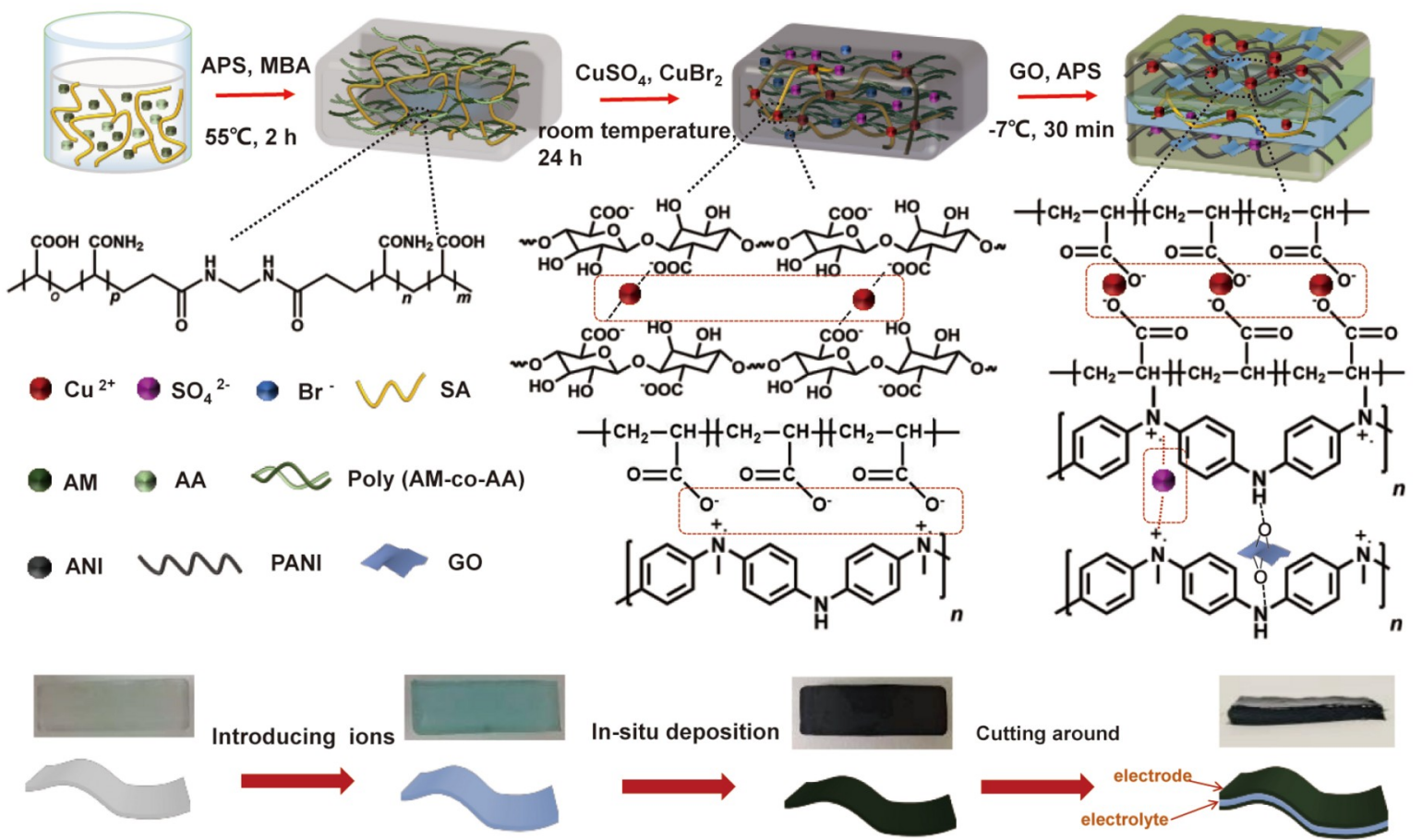

Figure 1 (a) Schematic and synthesis route of PANI/GO-GELE hydrogel complex. (b) Images (upper) and sketches (lower) of the assembly of the laminated AISC. 


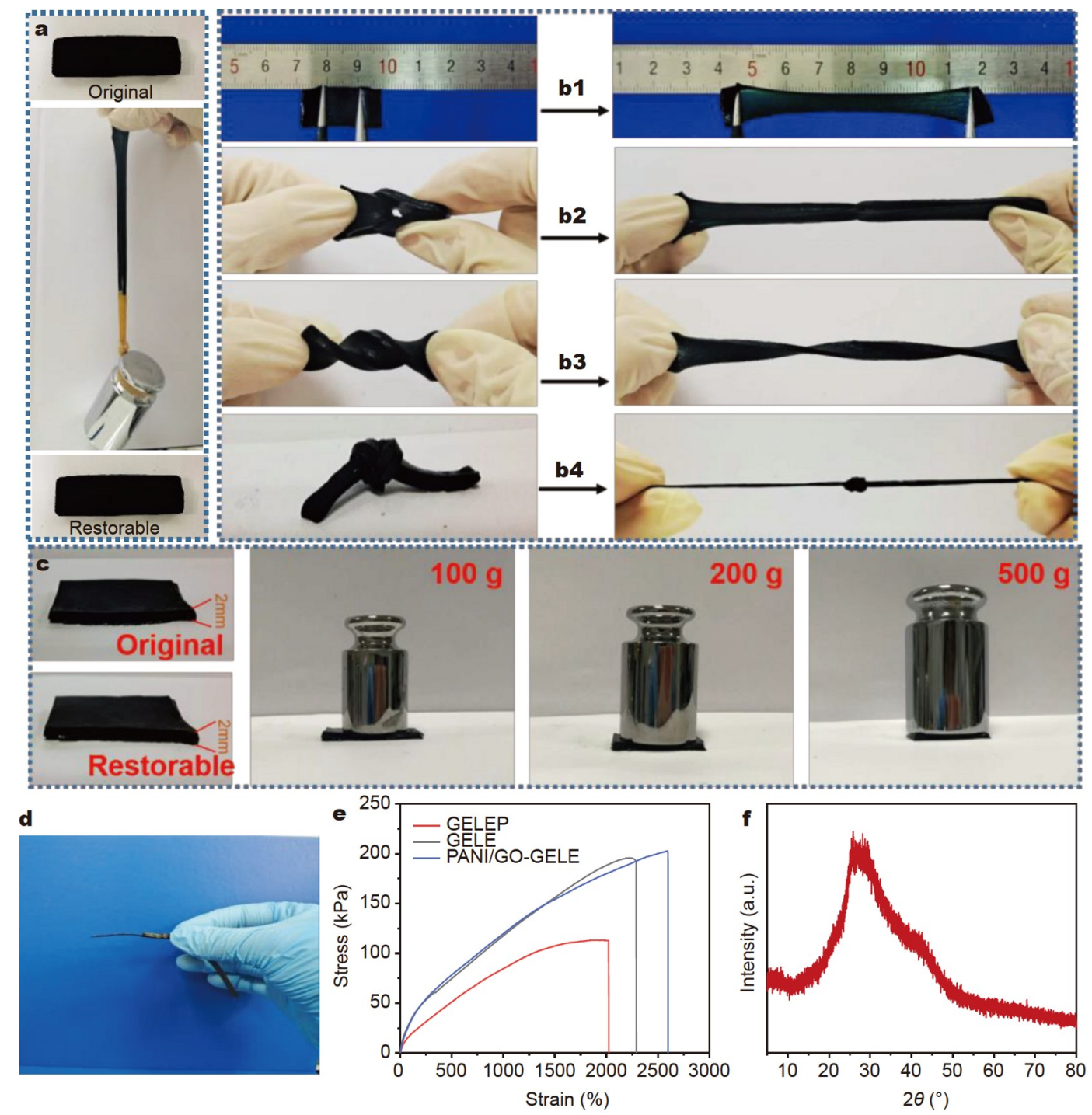

Figure 2 (a) PANI/GO-GELE hydrogel complex in sustaining a weight of $500 \mathrm{~g}$. (b1-b4) Bare, crossed, twisted, and knotted stretches for the hydrogel complex. (c) Compression properties of the hydrogel complex under weights of 100, 200, and $500 \mathrm{~g}$ and its shape recovery performance. (d) Adhesive behavior of PANI/GO-GELE hydrogel complex with carbon cloth. (e) Tensile stress-strain curves of different hydrogels. (f) XRD spectrum of PANI.

PANI, with the broad peak at $26^{\circ}$ indicating the successful polymerization and deposition of PANI in the hydrogel complex [26].

The microstructure of hydrogel substrates has been reported to exert a great influence on the electrochemical performance of integrated devices [27]. As shown in Fig. 3a, b, the inner morphology of the PANI/GO-GELE hydrogel complex was characterized by SEM after freeze-drying. The sponge-like hydrogel was uniformly distributed and porous. PANI nanofibers were entangled to form a conductive network across the planar hydrogel electrode. Pieces or small clusters of GO nanosheets were distinguished from the PANI networks in the enlarged images. The uniform dispersion of $\mathrm{Br}, \mathrm{Cu}, \mathrm{C}, \mathrm{O}$, and $\mathrm{S}$ were further proved by the EDS elemental mapping of the selected region shown in Fig. $3 \mathrm{c}-\mathrm{h}$ and confirmed the good incorporation of $\mathrm{Cu}^{2+}, \mathrm{SO}_{4}{ }^{2-}$, and $\mathrm{Br}^{-}$into GELE. In addition, the threedimensional (3D) porous laminated networks provided a sufficient attachment area for PANI growth. The channel structure improved the efficiency of mass transport, which further enhanced the electrochemical performance of the AISC device [28]. In general, the uniform distribution of the active material in the electrode layer, the highly conductive $3 \mathrm{D}$ polymer network, and the abundant active sites provided by the porous and thick PANI/GO matrix ensured excellent electrochemical performance in the integrated device [29].

\section{Electrochemical characterization of PANI/GO-GELE AISC}

The integrated SC was assembled for the electrochemical tests to determine the electrochemical properties of the PANI/GOGELE hydrogel complex. In the PANI/GO-GELE AISC system, the PANI/GO hydrogel was utilized as the electrode layer, the ion-interacted GELE film served as the solid electrolyte and separator, and the carbon cloth was adhered to the outer surface of the PANI/GO hydrogel and used as the current collector to form a solid-state energy storage device. Fig. 4a demonstrates the energy storage mechanism of the integrated device. The PANI stored energy through a redox process, which is detailed in Fig. S3. Ions were transferred to the conductive polymer elec- 

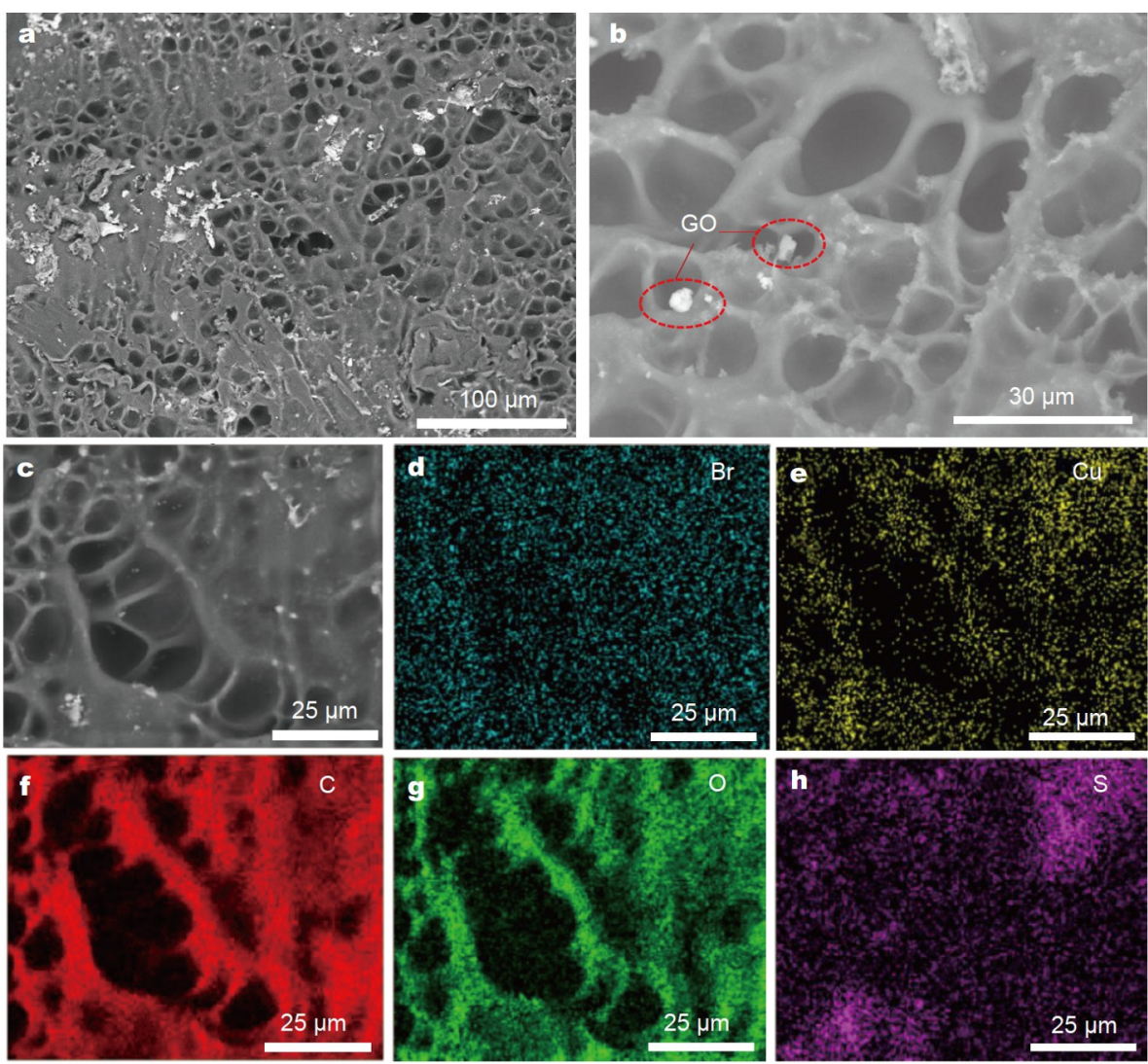

Figure 3 (a, b) SEM images of the PANI/GO-GELE hydrogel complex after freeze-drying and its partial enlargement. (c-h) EDS elemental mapping of a selected region.

trodes during the oxidation process and released from the entire backbone into the electrolytes during the reduction process. Moreover, the Faraday reaction of the bromide ions in the electrode/electrolyte interface could be directly combined with the electric double-layer electrode to promote additional energy storage [17]. The energy storage mechanism of bromide ions could be proved as follows:

$2 \mathrm{Br}^{-} \leftrightarrow \mathrm{Br}_{2}+2 \mathrm{e}^{-}$,

$\mathrm{Br}_{2}+\mathrm{Br}^{-} \leftrightarrow \mathrm{Br}_{3}^{-}$.

The serious electrode cracking during ion doping and purification is a major challenge affecting the electrochemical performance of the PANI electrodes [30]. Herein, the GO suspension was composited into the PANI network to improve the electrical conductivity and prevent the PANI network from cracking in the deprotonated state [31]. Fig. S4 compares the electrochemical performances of the AISC after the addition of $\mathrm{GO}$ on the basis of $\mathrm{CV}, \mathrm{GCD}$, and electrochemical impedance spectroscopy measurements. The PANI/GO electrode exhibited better specific capacitance and rate than the pure PANI electrode. For GELE, the multi-ion additives effectively increased the ion concentration in the electrolytes, thereby enabling rapid ion diffusion rates as a solid electrolyte to advance the rate performance of the AISC.

Fig. $4 \mathrm{~b}$ demonstrates the CV curves of the PANI/GO-GELE AISC at scan rates ranging from 10 to $100 \mathrm{mV} \mathrm{s}^{-1}$. The redox reaction process was observed through the $\mathrm{CV}$ curves, and the peak current and curve area gradually increased with the increase of the scan rates. This result is consistent with the pseudocapacitance characteristic of PANI. The GCD curves at different area current densities are shown in Fig. 4c. The specific capacitances were calculated to be $222.8,187.2,163.2,160.0$, and $151.7 \mathrm{mF} \mathrm{cm}^{-2}$ at $0.2,0.4,0.8,1.6$, and $3.2 \mathrm{~mA} \mathrm{~cm}^{-2}$, respectively, with the capacitance retentions being $84.0 \%, 73.2 \%, 71.8 \%$, and $68.1 \%$ (Fig. 4d). As shown in Fig. 4e, the capacitance retention for the PANI/GO-GELE AISC was $79.5 \%$ after 300 cycles. The Ragone plot calculated from the GCD curve is shown in Fig. 4f. The area energy densities of a piece of the device could reach $44.6,37.4,32.4,32.0$, and $30.34 \mu \mathrm{W} \mathrm{h} \mathrm{cm}{ }^{-2}$ at power densities of $120.0,240.0,476.4,960$, and $1919.6 \mu \mathrm{W} \mathrm{cm}{ }^{-2}$, respectively, thereby satisfying the energy density and power density required in full hydrogel-based flexible energy storage devices.

\section{Electrochemical performance of the PANI/GO-GELE AISC under mechanical deformation}

In the actual application of flexible electronics, the electrochemical performance of energy storage devices should be highly adaptable to complicated mechanical deformations under external stimuli [32].

Fig. 5a shows the testing assembly, in which one terminal of the SC was fixed by a small glass slide while the other terminal was rotated to control the bending angle. As demonstrated in Fig. 5b, the AISC showed excellent flexibility at bending angles of $30^{\circ}, 60^{\circ}, 90^{\circ} 120^{\circ}, 150^{\circ}$, and $180^{\circ}$. No misalignment or delamination of the electrode and electrolyte could be distinguished even under $180^{\circ}$ folding because of the in situ polymerization and quick deposition process of the active materials on the GELE film and the firm interfacial interaction in the sandwich 

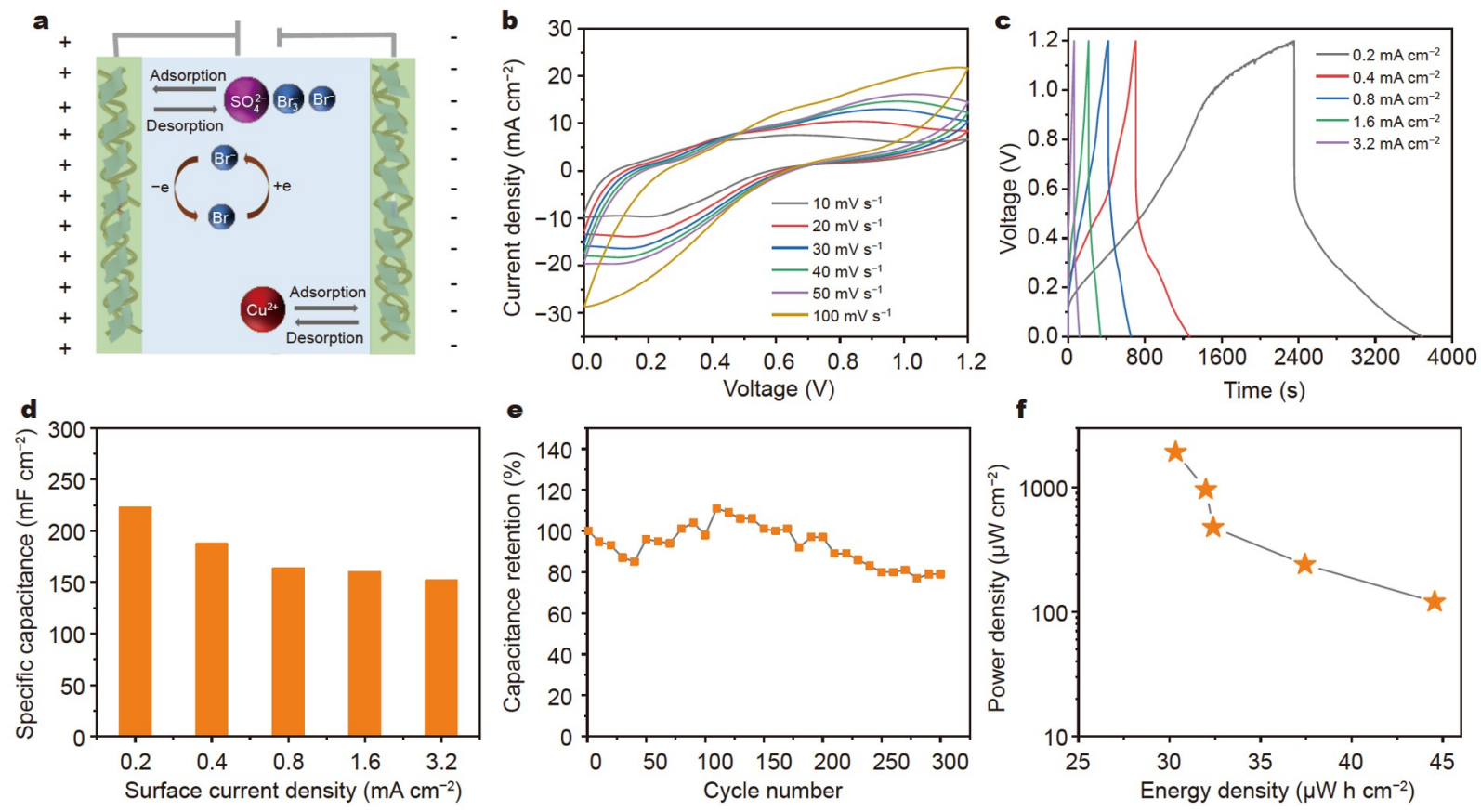

Figure 4 (a) Electrochemical energy storage mechanism of a PANI/GO-GELE AISC. (b) CV curves at different scan rates, (c) GCD curves at different current densities, (d) rate performance, (e) cycling stability at $3.2 \mathrm{~mA} \mathrm{~cm}^{-2}$, and (f) Ragone plots of the assembled AISC.
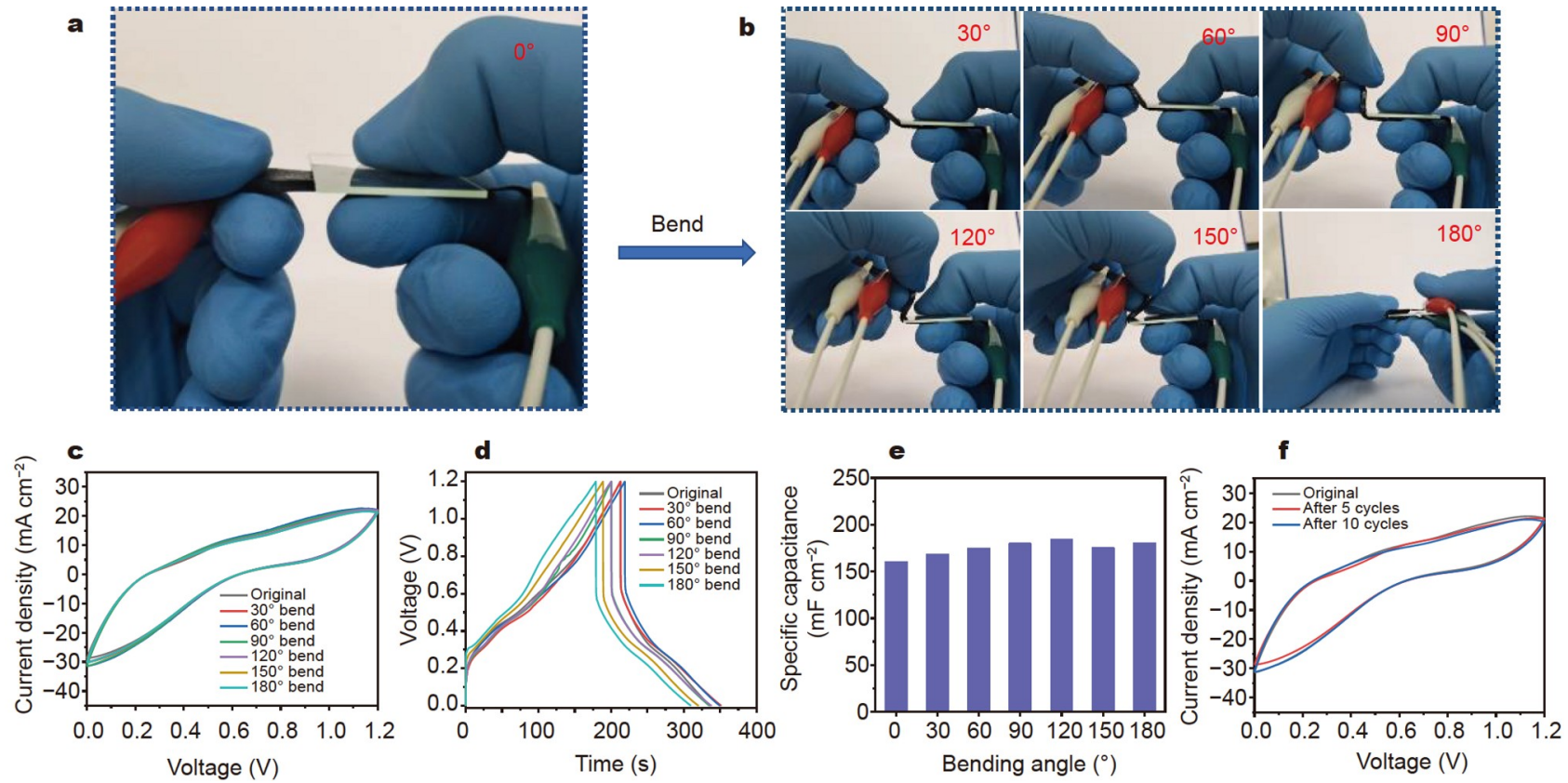

Figure 5 (a) Schematic of a PANI/GO-GELE AISC for bending tests. (b) PANI/GO-GELE AISC bending at different angles. (c) CV curves at $100 \mathrm{mV} \mathrm{s}^{-1}$, (d) GCD curves, and (e) calculated specific capacitances at $1.6 \mathrm{~mA} \mathrm{~cm}^{-2}$ for the PANI/GO-GELE AISC at different bending angles. (f) Consecutive CV cycles of the PANI/GO-GELE AISC bent at $90^{\circ}$ at $100 \mathrm{mV} \mathrm{s}^{-1}$.

structure. In the case of extreme bending at $180^{\circ}$, an almost negligible depression of the electrochemical performance was observed in the CV and GCD curves (Fig. $5 c$, d) and in the calculated specific capacitance (Fig. 5e). The slight increase of the specific capacitance might be attributed to the external force exerted on the device under bending. In addition, the electrochemical performance of the device showed high stability under bending cycles. As shown in Fig. 5f, the $\mathrm{CV}$ curves remained steady in 10 consecutive cycles under bending at $90^{\circ}$, thus indicating the AISC's excellent electrochemical performance in the bending tests.

Fig. 6a shows a diagram of the electrochemical tests of the device under pressures of $0,0.25,0.5,1,2.5$, and $5 \mathrm{kPa}$. The area enclosed by the CV curves in Fig. $6 \mathrm{~b}$ gradually increased with 


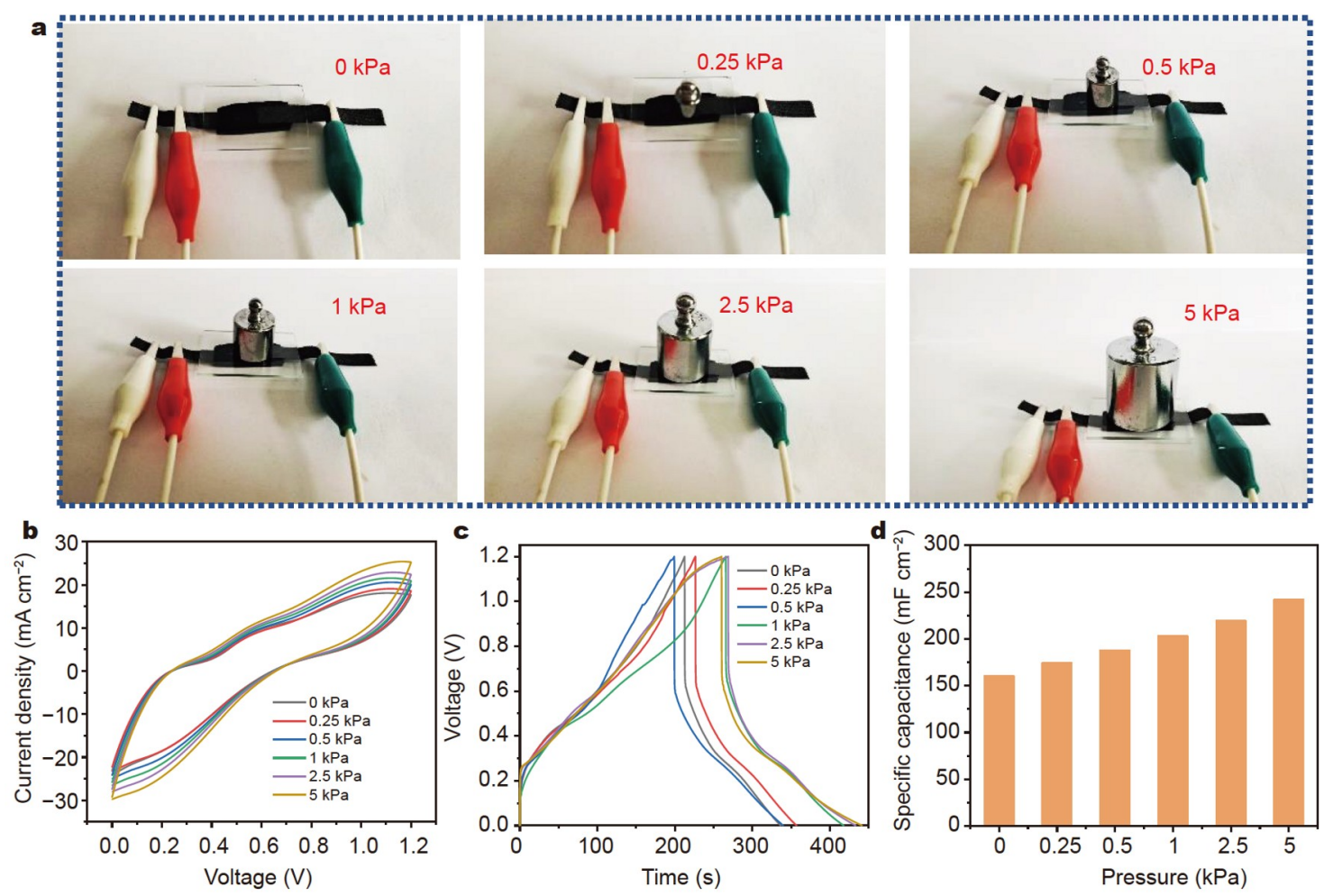

Figure 6 (a) PANI/GO-GELE AISC under pressures of $0,0.25,0.5,1,2.5$, and $5 \mathrm{kPa}$. (b) CV curves at $100 \mathrm{mV} \mathrm{s}^{-1}$, (c) GCD curves, and (d) calculated specific capacitances at $1.6 \mathrm{~mA} \mathrm{~cm}^{-2}$ of the PANI/GO-GELE AISC under different pressures.

the increase of pressure; such a result was consistent with the GCD curves in Fig. $6 c$ and the calculated specific capacitance in Fig. $6 \mathrm{~d}$. Under compression at $5 \mathrm{kPa}$, the specific capacitance exceeded nearly $50 \%$ of that of the original device. The literature [33] shows that the adhesion of hydrogels is weakened under compression and that the adhesion property of polymers is inversely proportional to conductivity. Hence, ionic conductivity would apparently increase under compression. With an acceleration of the ion transmission rate at the electrode/electrolyte interface, the electrochemical performance of the integrated devices could be greatly enhanced. In addition, because of the high elasticity of hydrogels, the increase of external compression could shorten the ion diffusion distance to accelerate the redox reaction [34]. Furthermore, increasing compression could strengthen the interfacial contact between electrodes and electrolytes and thereby increase the accessible electrochemical active sites and accelerate charge transfer [35]. Such conjecture could be verified by the decrease of the internal resistance of the device under compression in Fig. S5. Therefore, the integrated AISC could display comparable or even enhanced energy storage performance through the introduction of compression.

The rapid development of flexible energy storage devices for practical applications is required to ensure excellent energy output under mechanical stretching. Fig. 7a shows a schematic of the AISC device at tensile strains of $25 \%$ and $50 \%$, which are adequate for most types of human motion detection mechanisms in wearable devices. As shown by the CV curves in Fig. 7b, the enclosed area decreased with increasing tensile strains. Calculated from the GCD curves in Fig. 7c, the specific capacitances decreased to 114.9 and $94.5 \mathrm{mF} \mathrm{cm}^{-2}$ with strains of $25 \%$ and $50 \%$, respectively (Fig. $7 \mathrm{~d}$ ). Hence, the GELE film with multiple polymer crosslinking networks ensured the excellent toughness and mechanical strength of the hydrogel complex, inhibited the generation of microcracks on the PANI/GO hydrogel electrode during stretching, maintained the stability of the conductive network, and sustained the reliable electrochemical performance of the PANI/GO-GELE AISC device. These results indicate that the integrated SCs in the antideformation tests could meet practical requirements [36].

The working voltage window was tested to further increase the energy density of stretchable SCs and expand the practical applications of the PANI/GO-GELE AISC. As shown in Fig. 8a, the operating voltage window could reach $1.5 \mathrm{~V}$ without severe polarization; the result is higher than that obtained with most integrated, flexible SCs $[16,28,37]$. Fig. 8 b also shows convincing electrochemical performance in the CV curves under cycling at different scan rates. The expanded voltage window was attributed to a typical amphiphilic structure of the hydrogel electrolyte rich in multiple ions. The strong interaction between the abundant charged groups and the water molecules (76.0\%) resulted in the expansion of hydrogen and oxygen evolution voltage [38]. As demonstrated in Fig. 8c, three pieces of AISCs could be easily connected in series owing to their inherently strong adhesive property, and the fully charged prototype could power a red LED light with steady energy output.

\section{CONCLUSIONS}

The integrated PANI/GO-GELE hydrogel complex was prepared into a sandwiched structure through a facile method. The assembled PANI/GO-GELE AISC showed excellent electrochemical performance with specific capacitances of 222.8, 187.2, 163.2, 160.0, and $151.7 \mathrm{mF} \mathrm{cm}^{-2}$ at $0.2,0.4,0.8,1.6$, and 

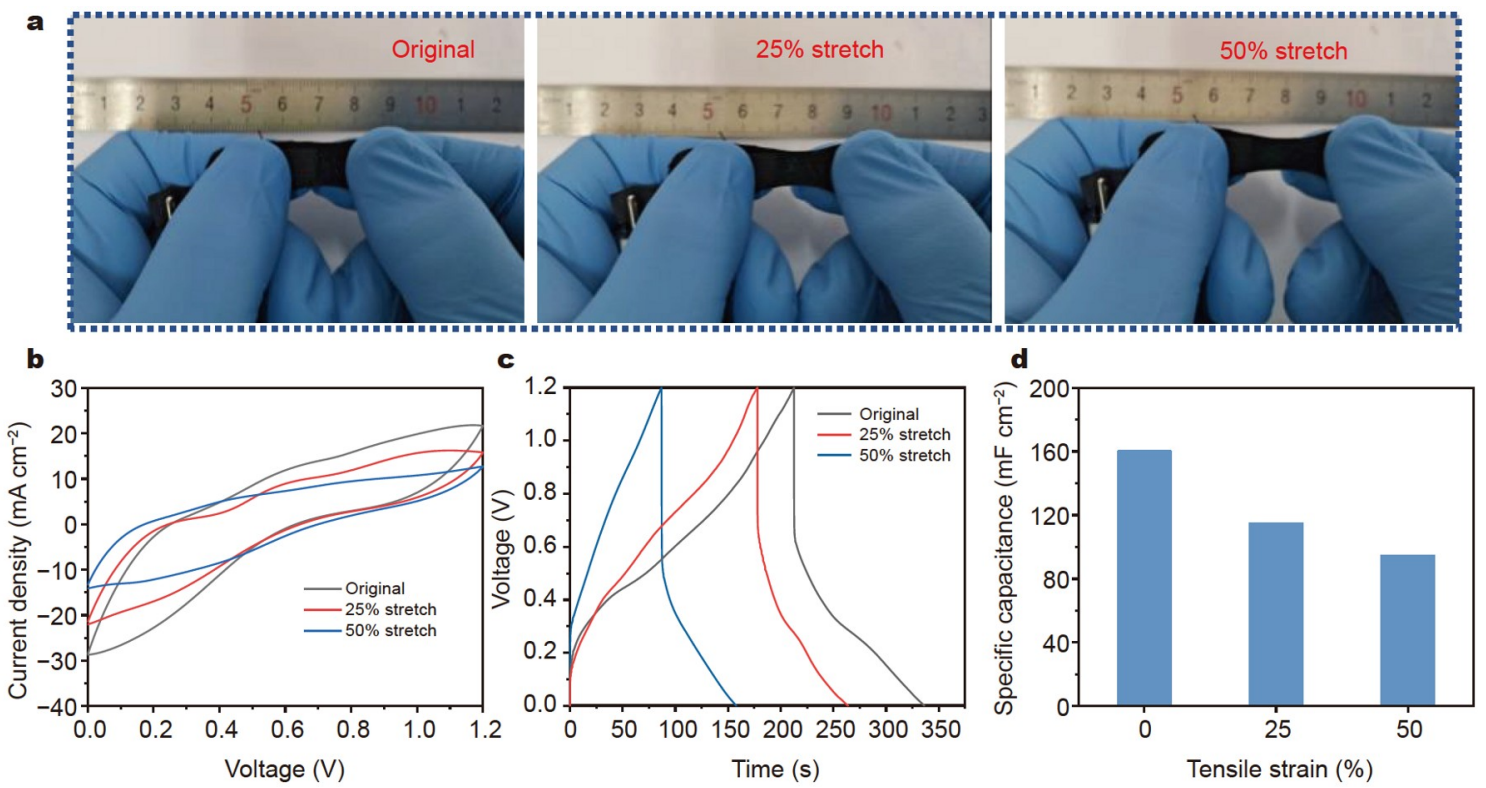

Figure 7 (a) Schematic of PANI/GO-GELE AISC under different tensile strains. (b) CV curves at $100 \mathrm{mV} \mathrm{s}^{-1}$, (c) GCD curves, and (d) calculated specific capacitances at $1.6 \mathrm{~mA} \mathrm{~cm}^{-2}$ for PANI/GO-GELE AISC under different tensile strains.
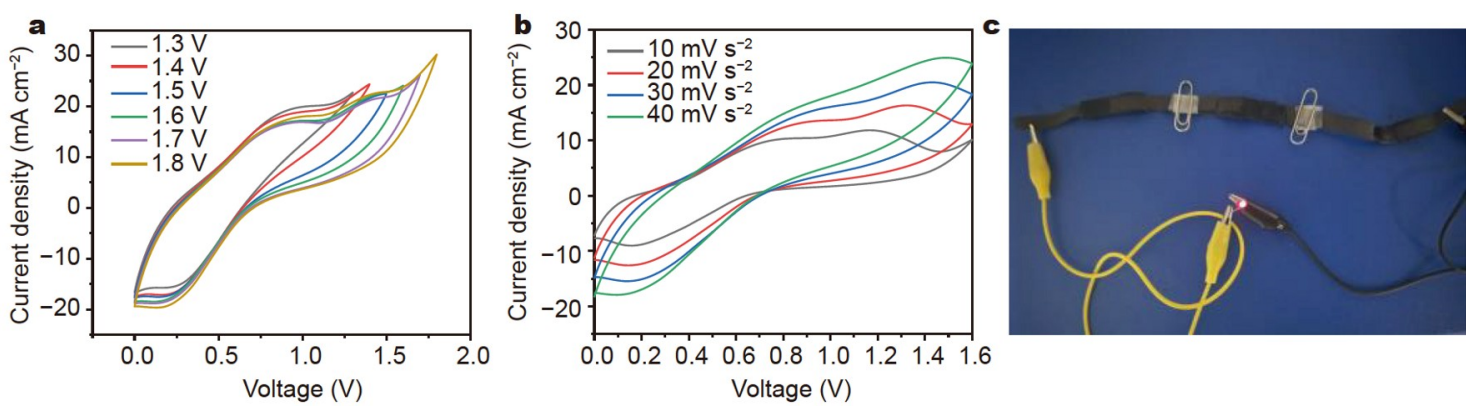

Figure 8 (a) CV curves of the PANI/GO-GELE AISC in different voltage windows at $50 \mathrm{mV} \mathrm{s}^{-1}$. (b) CV curves of the PANI/GO-GELE AISC at different scan rates in the voltage range of $0-1.6 \mathrm{~V}$. (c) Diagram of three pieces of AISCs in series connection to power LED light.

$3.2 \mathrm{~mA} \mathrm{~cm}^{-2}$, respectively. It also displayed a superior energy density of $44.6 \mu \mathrm{W} \mathrm{h} \mathrm{cm}{ }^{-2}$ at a power density of $120.0 \mu \mathrm{W} \mathrm{cm}$. Moreover, the AISCs revealed excellent mechanical deformation resistance to bending, compression, and stretching while maintaining reliable and reproducible electrochemical performance. Owing to the strong adhesion property of the hydrogel complex, the AISCs could be connected in series to power LED lights and thus demonstrate practical application prospects. The proposed PANI/GO-GELE AISC with remarkable electrochemical performances under mechanical deformation provides a novel approach to the development of flexible energy storage devices.

\section{Received 15 May 2021; accepted 29 July 2021;}

published online 10 September 2021

1 Chen CR, Qin H, Cong HP, et al. A highly stretchable and real-time healable supercapacitor. Adv Mater, 2019, 31: 1900573

2 Zhao W, Jiang M, Wang W, et al. Flexible transparent supercapacitors: Materials and devices. Adv Funct Mater, 2020, 31: 2009136

3 Li T, Fang X, Pang Q, et al. Healable and shape editable supercapacitors based on shape memory polyurethanes. J Mater Chem A, 2019, 7: 17456-17465

4 Eftekhari A, Li L, Yang Y. Polyaniline supercapacitors. J Power Sources,
2017, 347: 86-107

5 Yao B, Wang $\mathrm{H}$, Zhou Q, et al. Ultrahigh-conductivity polymer hydrogels with arbitrary structures. Adv Mater, 2017, 29: 1700974

6 Sharma P, Bhatti TS. A review on electrochemical double-layer capacitors. Energy Convers Manage, 2010, 51: 2901-2912

7 Oh J, Kim YK, Lee JS, et al. Highly porous structured polyaniline nanocomposites for scalable and flexible high-performance supercapacitors. Nanoscale, 2019, 11: 6462-6470

8 Wang Z, Zhu M, Pei Z, et al. Polymers for supercapacitors: Boosting the development of the flexible and wearable energy storage. Mater Sci Eng-R-Rep, 2020, 139: 100520

9 Zeng S, Chen H, Cai F, et al. Electrochemical fabrication of carbon nanotube/polyaniline hydrogel film for all-solid-state flexible supercapacitor with high areal capacitance. J Mater Chem A, 2015, 3: 2386423870

10 Chen J, Peng Q, Thundat $\mathrm{T}$, et al. Stretchable, injectable, and selfhealing conductive hydrogel enabled by multiple hydrogen bonding toward wearable electronics. Chem Mater, 2019, 31: 4553-4563

11 Huang Y, Zhong M, Huang Y, et al. A self-healable and highly stretchable supercapacitor based on a dual crosslinked polyelectrolyte. Nat Commun, 2015, 6: 10310

12 Yang Z, Ma J, Bai B, et al. Free-standing PEDOT/polyaniline conductive polymer hydrogel for flexible solid-state supercapacitors. Electrochim Acta, 2019, 322: 134769

13 Nan J, Zhang G, Zhu T, et al. A highly elastic and fatigue-resistant 
natural protein-reinforced hydrogel electrolyte for reversible-compressible quasi-solid-state supercapacitors. Adv Sci, 2020, 7: 2000587

14 Zhao T, Ji X, Bi P, et al. In situ synthesis of interlinked three-dimensional graphene foam/polyaniline nanorod supercapacitor. Electrochim Acta, 2017, 230: 342-349

15 Hu M, Wang J, Liu J, et al. An intrinsically compressible and stretchable all-in-one configured supercapacitor. Chem Commun, 2018, 54: 6200-6203

16 Guo Y, Zheng K, Wan P. A flexible stretchable hydrogel electrolyte for healable all-in-one configured supercapacitors. Small, 2018, 14: 1704497

17 Han L, Huang H, Li J, et al. A novel redox bromide-ion additive hydrogel electrolyte for flexible $\mathrm{Zn}$-ion hybrid supercapacitors with boosted energy density and controllable zinc deposition. J Mater Chem A, 2020, 8: 15042-15050

18 Gao Z, Zhang L, Chang J, et al. Catalytic electrode-redox electrolyte supercapacitor system with enhanced capacitive performance. Chem Eng J, 2018, 335: 590-599

19 Zhou X, Ren Y, Lu Y, et al. Porous Mo-Co-S nanosheets on carbon cloth for all-solid-state flexible asymmetric supercapacitors. Adv Mater Interfaces, 2019, 6: 1901138

20 Ren Y, Du H, Zhou X, et al. Three-dimensional Al-substituted quasiconcentration gradient $\mathrm{Ni}$-Co layered double hydroxide nanosheets for high-performance asymmetric supercapacitors. Mater Today Energy, 2020, 18: 100514

21 Gong Z, Zhang G, Zeng X, et al. High-strength, tough, fatigue resistant, and self-healing hydrogel based on dual physically cross-linked network. ACS Appl Mater Interfaces, 2016, 8: 24030-24037

22 Xia Y, Wang Z, Chen LY, et al. Nanoscale polyelectrolyte/metal ion hydrogel modified RO membrane with dual anti-fouling mechanism and superhigh transport property. Desalination, 2020, 488: 114510

23 Ge G, Zhang Y, Shao J, et al. Stretchable, transparent, and selfpatterned hydrogel-based pressure sensor for human motions detection. Adv Funct Mater, 2018, 28: 1802576

24 Wang M, Yang J, Liu S, et al. Nitrogen-doped hierarchically porous carbon nanosheets derived from polymer/graphene oxide hydrogels for high-performance supercapacitors. J Colloid Interface Sci, 2020, 560: 69-76

25 Chen W, Jiang S, Xiao H, et al. Graphene modified polyanilinehydrogel based stretchable supercapacitor with high capacitance and excellent stretching stability. ChemSusChem, 2021, 14: 938-945

26 Bhowmik KL, Deb K, Bera A, et al. Charge transport through polyaniline incorporated electrically conducting functional paper. J Phys Chem C, 2016, 120: 5855-5860

27 Hsu HH, Liu Y, Wang Y, et al. Mussel-inspired autonomously selfhealable all-in-one supercapacitor with biocompatible hydrogel. ACS Sustain Chem Eng, 2020, 8: 6935-6948

28 Zhao Y, Zhang B, Yao B, et al. Hierarchically structured stretchable conductive hydrogels for high-performance wearable strain sensors and supercapacitors. Matter, 2020, 3: 1196-1210

29 Zhang G, Chen Y, Deng Y, et al. A triblock copolymer design leads to robust hybrid hydrogels for high-performance flexible supercapacitors. ACS Appl Mater Interfaces, 2017, 9: 36301-36310

30 Afif A, Rahman SM, Tasfiah Azad A, et al. Advanced materials and technologies for hybrid supercapacitors for energy storage-A review. J Energy Storage, 2019, 25: 100852

31 Luo J, Zhong W, Zou Y, et al. Preparation of morphology-controllable polyaniline and polyaniline/graphene hydrogels for high performance binder-free supercapacitor electrodes. J Power Sources, 2016, 319: 7381

32 Ge G, Cai Y, Dong Q, et al. A flexible pressure sensor based on rGO/ polyaniline wrapped sponge with tunable sensitivity for human motion detection. Nanoscale, 2018, 10: 10033-10040

33 Masarapu C, Wang LP, Li X, et al. Tailoring electrode/electrolyte interfacial properties in flexible supercapacitors by applying pressure. Adv Energy Mater, 2012, 2: 546-552

34 Liu X, Wu D, Wang $\mathrm{H}$, et al. Self-recovering tough gel electrolyte with adjustable supercapacitor performance. Adv Mater, 2014, 26: 43704375
35 Scott MP, Rahman M, Brazel CS. Application of ionic liquids as lowvolatility plasticizers for PMMA. Eur Polym J, 2003, 39: 1947-1953

36 Yang J, Yu X, Sun X, et al. Polyaniline-decorated supramolecular hydrogel with tough, fatigue-resistant, and self-healable performances for all-in-one flexible supercapacitors. ACS Appl Mater Interfaces, 2020, 12: $9736-9745$

37 Lou D, Wang C, He Z, et al. Robust organohydrogel with flexibility and conductivity across the freezing and boiling temperatures of water. Chem Commun, 2019, 55: 8422-8425

38 Lei $\mathrm{Z}, \mathrm{Wu}$ P. Zwitterionic skins with a wide scope of customizable functionalities. ACS Nano, 2018, 12: 12860-12868

Acknowledgements This work was supported by the Natural Science Foundation of Shandong Province (ZR2020KB018), the Natural Science Foundation of Jiangsu Province (BK20190688), the Six Talent Peak Innovation Team in Jiangsu Province (TD-SWYY-009), the "Taishan Scholars" Construction Special Fund of Shandong Province, and the Industrial Alliance Fund of Shandong Provincial Key Laboratory (SDKL2016038).

Author contributions Wang $\mathrm{Q}$ and Ren $\mathrm{Y}$ designed the project and experiments. Ren Y, Sun C, Hong Y, Li S, Zhao W, and Liu Y prepared and characterized the materials. Ren Y wrote the manuscript with the support from Dong X. All authors contributed to the data analyses, discussed the results, and commented on the manuscript.

Conflict of interest The authors declare that they have no conflict of interest.

Supplementary information Supporting data are available in the online version of the paper.

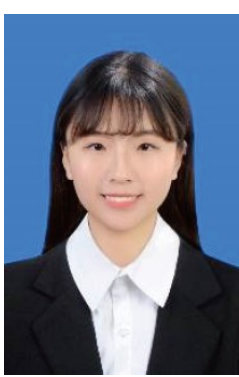

Yanfang Ren is a master candidate at Liaocheng University under the supervision of Prof. Wenjun Wang. Currently, she is studying at Nanjing Tech University as an exchange student in Prof. Xiaochen Dong's team. Her research interests include energy materials and flexible electronics.

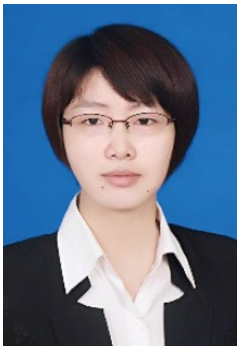

Qian Wang received her $\mathrm{PhD}$ degree from the University of Chinese Academy of Sciences in 2015. Then, she continued her postdoctoral research at Shanghai Institute of Microsystem and Information Technology, Chinese Academy of Sciences, for three years. Her current research interest is focused on the design and preparation of nanomaterials for flexible electronics.

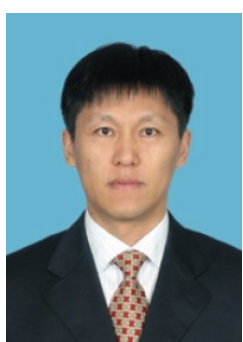

Xiaochen Dong earned his PhD degree from Zhejiang University in 2007. He completed his postdoctoral research at Nanyang Technological University, Singapore. In 2012, he joined Nanjing Tech University as a full professor. His research interest is nanomaterials for bio-optoelectronics and energy conversion and storage. 


\section{原位沉积多层集成水凝胶用于可变形拉伸超级电容器}

任燕芳 ${ }^{1}$, 孙陈诚 ${ }^{3}$, 刘云龙 ${ }^{1}$, 洪颢 ${ }^{2}$, 王倩 $2^{*}$, 赵雯丽 ${ }^{2}$, 李淑红 ${ }^{*}$, 王文军 ${ }^{1}$, 董晓卧

摘要 因其固有的机械弹性和离子电导率, 水凝胶体系极大地促进了 柔性储能设备的发展. 然而, 对于水凝胶装置而言, 在剧烈的机械变形 下实现稳定的能量存储仍然是一个挑战. 本文通过在离子结合水凝胶 电解质的两侧原位沉积聚苯胺/氧化石墨烯(PANI/GO)纳米复合材料, 组装一体化集成超级电容器(AISC), 简化了器件组装过程, 并避免了水 凝胶储能器件在机械变形过程中多层结构的置换和分离; 同时, 离子添 加剂水凝胶电解质表现出较强的粘附性、柔韧性以及高离子电导率, 确保了AISC优异的比电容和倍率性能. 其在 $0.2 \mathrm{~mA} \mathrm{~cm}^{-2}$ 电流密度下比 电容为 $222.8 \mathrm{~m} \mathrm{~F} \mathrm{c} \mathrm{m}^{-2}$, 在 $3.2 \mathrm{~m} \mathrm{~A} \mathrm{c} \mathrm{m}^{-2}$ 电流密度下比电容为 $151.7 \mathrm{mF} \mathrm{cm}^{-2}$, 电容保持率为 $68.1 \%$. 器件在 $120.0 \mu \mathrm{W} \mathrm{cm}-2$ 的功率密度 下, 能量密度达到 $44.6 \mu \mathrm{W} \mathrm{h} \mathrm{cm}{ }^{-2}$. 此外, 在弯曲、压缩和拉伸的变形下 可以获得稳定的能量存储, 串联组装的AISC可以为LED灯供电. 该工作 在改善超级电容器机械性能的同时进一步提高了其电学性能, 为构建 新型柔性超级电容器提供了一种新策略. 